\title{
Endocrine referral system audit and quality improvement project
}

\author{
Authors: Kate Millar, Alex Stevenson and Sheharyar Qureshi
}

\section{Aims}

Audit of current endocrine referral system in a busy district general hospital with a view to:

> improving efficacy and minimising time delay by development of an online referral system

> subsequent re-audit of this system to enhance quality and multidisciplinary team care

> identify areas for service gap and further improvement based on user feedback.

\section{Methods}

Original paper based referral system audit was undertaken over 4 weeks to ascertain referral to specialty review time for all in patient diabetes and endocrine referrals. This confirmed delay in response time to referrals and a poor use of the specialty service. An online referral form was developed within the existing Integrated Clinical Environment (ICE) request system. This allowed referrals to be requested and received by the endocrine specialist registrars, diabetic specialist nurses and diabetic foot service from any computer in the hospital. An audit trail could also be followed to identify referrals that were not been acted on within the ideal time frame (with 24 hours). This online referral system was then reaudited 12 months following the original audit and data analysed to enhanced quality of endocrine specialty services.

\section{Results}

The original paper based system only collected data from eight referral requests; of which three $(37.5 \%)$ were answered over 48 hours after the request and only two $(25 \%)$ in less than 6 hours. In comparison, within 6 months of introduction of the online system the team received 90 referrals over the same one-month period. Of these $67(74.44 \%)$ were responded to within the target 24 hours, with $47(52.22 \%)$ receiving a specialty response within 6 hours. However, $13(14.44 \%)$ were still responded to in over 48 hours.

\section{Conclusion}

Switching referrals from paper based to electronic (via ICE) has dramatically improved timely access to specialist diabetes/ endocrine advice for medical teams at West Middlesex University
Hospital. Total number of referrals to our service increased and efficacy of response to review time has improved.

We have identified further area of improvement ie some referrals that are not meeting the 24 hour target review time but mostly the delay was secondary to out of hour use of above system. We have agreed to enhance this system based on service users and warrant further hospital based services to use online platforms with a view to enhance the quality of their service.

\section{Conflict of interest statement}

None declared 